\title{
Early White Matter Changes in Childhood Multiple Sclerosis: A Diffusion Tensor Imaging Study
}

\author{
A. Blaschek, D. Keeser, S. Müller, I.K. Koerte, A. Sebastian Schröder, W. Müller-Felber, F. Heinen, and B. Ertl-Wagner
}

\begin{abstract}
BACKGROUND AND PURPOSE: Loss of integrity in nonlesional white matter occurs as a fundamental feature of multiple sclerosis in adults. The purpose of our study was to evaluate DTI-derived measures of white matter microstructure in children with MS compared with age- and sex-matched controls by using tract-based spatial statistics.
\end{abstract}

MATERIALS AND METHODS: Fourteen consecutive pediatric patients with MS (11 female/3 male; mean age, $15.1 \pm 1.6$ years; age range, 12-17 years) and age- and sex-matched healthy subjects (11 female/3 male; mean age, $14.8 \pm 1.7$ years) were included in the study. After we obtained DTI sequences, data processing was performed by using tract-based spatial statistics.

RESULTS: Compared with healthy age- and sex-matched controls, children with multiple sclerosis showed a global decrease in mean fractional anisotropy $(P \leq .001)$, with a concomitant increase in mean $(P<.001)$, radial $(P<.05)$, and axial diffusivity $(P<.001)$. The most pronounced fractional anisotropy value decrease in patients with MS was found in the splenium of the corpus callosum $(P<.001)$. An additional decrease in fractional anisotropy was identified in the right temporal and right and left parietal regions $(P<.001)$. Fractional anisotropy of the white matter skeleton was related to disease duration and may, therefore, serve as a diagnostic marker.

CONCLUSIONS: The microstructure of white matter is altered early in the disease course in childhood multiple sclerosis.

ABBREVIATIONS: $A D=$ axial diffusivity; $F A=$ fractional anisotropy; $M D=$ mean diffusivity; $R D=$ radial diffusivity; TBSS $=$ tract-based spatial statistics

M S most commonly occurs in adults in their late 20 s and 30 s, but early onset in childhood and adolescence is increasingly being diagnosed, with up to $10 \%$ of MS cases manifesting before adulthood. Conventional MR imaging has become an essential part of diagnostic decision making in MS. On MR imaging, children tend to have fewer white matter lesions and less enhance-

Received November 15, 2012; accepted after revision January 3, 2013.

From the Department of Pediatric Neurology and Developmental Medicine (A.B., A.S.S., W.M.-F., F.H.), Hauner Children's Hospital, Ludwig-Maximilian-University, Munich, Germany; Department of Psychiatry and Psychotherapy (D.K.) and Institute for Clinical Radiology (D.K., S.M., I.K.K., B.E.-W.), Ludwig-Maximilian University, Munich, Germany; and Psychiatry Neuroimaging Laboratory (I.K.K.), Brigham and Women's Hospital, Harvard Medical School, Boston, Massachusetts.

A. Blaschek and D. Keeser contributed equally to the manuscript. F. Heinen and B. Ertl-Wagner shared senior authorship.

This work was supported by a research grant from Merck Serono. Inga Koerte was supported by the Else Kröner-Fresenius Stiftung, Bad Homburg, Germany.

Please address correspondence to Astrid Blaschek, MD, Department of Pediatric Neurology and Developmental Medicine, Hauner Children's Hospital, LudwigMaximilian-University Munich, Lindwurmstraße 4, 80337 Munich, Germany; e-mail: astrid.blaschek@med.uni-muenchen.de

$\equiv$ Indicates article with supplemental on-line tables.

Indicates article with supplemental on-line figure.

Evidence-Based Medicine Level 2.

http://dx.doi.org/10.3174/ajnr.A3581 ment with gadolinium-based contrast agents. ${ }^{1}$ Lesion load has been shown to correlate moderately with clinical outcome in longitudinal studies in children and adults. ${ }^{2}$

In addition to the above-mentioned localized lesions, white matter microstructure is known to be altered in the macroscopically normal-appearing white matter. ${ }^{3}$ Advanced MR imaging techniques such as DTI allow the evaluation of the microstructure of the cerebral white matter by detecting subtle changes in the magnitude and direction of water diffusion. White matter damage is mainly reflected by a decrease in fractional anisotropy (FA) and an increase in mean diffusivity (MD). MD consists of axial diffusivity $(\mathrm{AD})$ and radial diffusivity $(\mathrm{RD})$, measuring diffusivity parallel and perpendicular to the main axis of white matter tracts, respectively. ${ }^{4}$ Both parameters are purported to indicate myelin and axonal injury. ${ }^{5}$ Recently, DTI studies in adult patients with MS demonstrated axonal and myelin injury occurring much earlier in the course of the disease than previously assumed. ${ }^{6,7}$

Physiologic myelination is known to expand well into early adulthood in conjunction with cognitive, behavioral, emotional, and motor development. ${ }^{8-10}$ Any pathology interfering with this process of myelination is likely to affect white matter integrity as has been shown in hypoxic-ischemic encephalopathy, former premature in- 
fants, and normal-pressure hydrocephalus. ${ }^{11}$ The onset of childhood MS occurs within this vulnerable period of central nervous system maturation. To date, only a few MR imaging studies have investigated white matter microstructure in children with MS. These studies demonstrated decreased $\mathrm{FA}^{12}$ or slightly increased mean diffusivity ${ }^{13}$ in the macroscopically unaffected white matter by using either summary measures of the entire brain or a region-of-interest approach. Most recently, a study assessing major white matter tracts in children with MS compared with children with a single demyelinating event showed a decrease in FA with a concomitant increase in MD in patients with MS only. ${ }^{14,15}$

Tract-based spatial statistics (TBSS) allows a coregistration of likely white matter tracts to analyze multisubject diffusion tensor data. For this purpose, subject data are projected on a mean FA skeleton, which refers to the center of all tracts common to both groups, before applying voxelwise between-group comparisons. ${ }^{16}$ This technique permits an observer-independent voxelwise analysis of the main white matter tracts with a sensitivity to intergroup differences, considering less across-subject FA variability. ${ }^{16}$

The aim of our study was to evaluate DTI-derived measures of white matter microstructure in children with MS compared with age- and sex-matched controls by using TBSS.

\section{MATERIALS AND METHODS}

\section{Subjects}

This study was approved by the local institutional review board. Oral and written informed consent was obtained from all participants and their legal guardians.

Fourteen consecutive pediatric patients with MS ( 11 female/3 male; mean age, $15.1 \pm 1.6$ years; age range, $12-17$ years) were included in the study. Inclusion criteria were an age younger than 18 years and a definite diagnosis of MS with a relapsing-remitting course. Exclusion criteria consisted of MR imaging-related contraindications (eg, cardiac pacemakers, ferromagnetic implants, or claustrophobia). All patients were clinically evaluated on the basis of the Kurtzke Expanded Disability Status Scale.

MR imaging of age- and sex-matched healthy subjects (11 female/ 3 male; mean age, $14.8 \pm 1.7$ years) was included from a local data base of healthy volunteers; all volunteers had been imaged with the identical MR imaging protocol on the same MR imaging scanner (Magnetom Verio; Siemens, Erlangen, Germany). All volunteers underwent MR imaging for scientific purposes only. Inclusion criteria were an age match to a patient included in the MS cohort of \pm 6 months and a sex match to the respective patient. Exclusion criteria were any history of chronic or ongoing medical conditions, intake of medication, a history of traumatic brain injury (including mild traumatic brain injury), a history of headache disorders, a history of learning disorders, any history of other neurologic or psychiatric disorders, and structural brain abnormalities on conventional MR imaging sequences.

\section{Clinical Tests}

As part of the clinical work-up, selected elements of standardized tests were available for 13 of the 14 patients. Two parts of the Multiple Sclerosis Functional Composite score were administered: the right- and left-hand Nine-Hole Peg Test, to measure upper extremity fine motor skills, and the Timed 25-Foot Walk
Test, to assess the lower extremities. All values were compared with normative data. ${ }^{17,18}$ All clinical scores were converted to $z$ scores. The $z$ score indicates the deviation from the mean population score. A $z$ score lying outside the $95 \%$ normal distribution is considered abnormal. Screening of cognitive function was performed with 2 subtests of widely used cognitive tests in 11 patients: the Trail-Making Test (subtests A and B) ${ }^{19}$ and the German Leistungsprüfungssystem (subtest 5) for verbal fluency. ${ }^{20}$ All raw values were age-corrected for each patient according to Helmstaedter et al, ${ }^{21}$ to receive an age-independent measure.

\section{MR Imaging Acquisition}

MR imaging of the brain was performed on a 3T scanner (Magnetom Verio; Siemens Healthcare, Erlangen, Germany) by using a 12-element phased-array head coil for both the patient cohort and the healthy control subjects. The following structural sequences were acquired for all subjects: 3D magnetization-prepared rapid acquisition of gradient echo: TR, $11 \mathrm{~ms}$; TE, $4.76 \mathrm{~ms}$; FOV, $250 \mathrm{~mm}$; voxel size, $1 \times 1 \times 1 \mathrm{~mm}^{3}$; iPAT (Siemens syngo software) acceleration factor, 2; sagittal sections covering the entire brain, 160; and FLAIR: TR, 94 $\mathrm{ms}$; TE, $7000 \mathrm{~ms}$; FOV, $220 \mathrm{~mm}$; voxel size, $0.9 \times 0.9 \times 3 \mathrm{~mm}^{3}$; distance factor, $10 \%$; fat saturation; axial sections, covering the entire brain, 45. A DTI sequence with 20 independent diffusion directions and 3 averages was applied with the following parameters: $b=0$ and $1000 \mathrm{~s} / \mathrm{mm}^{2}$; matrix size, $128 \times 128 \mathrm{~mm}^{2}$; FOV, $230 \times 230 \mathrm{~mm}^{2}$. The resulting voxel size was $1.8 \times 1.8 \times 4.0 \mathrm{~mm}^{3}$. Thirty-six transverse sections were acquired.

\section{Image Analysis and Postprocessing}

Evaluation of Lesions. All structural sequences were visually assessed and graded by a board-certified neuroradiologist with $>10$ years' experience in MR imaging of the brain.

DTI Analysis. Image data processing was performed by using the TBSS approach implemented in FMRIB Software Library 4.19 (FSL; http://www.fmrib.ox.ac.uk/fsl). Images were corrected for eddy currents due to changing gradient fields and head motion. ${ }^{22}$ Brain masks were created by using the Brain Extraction Tool in FSL. ${ }^{22}$ FA and mean, axial, and radial diffusivity were calculated for each voxel. FA data of all subjects were aligned to a common space by using the FMRIB58 FA standard space with nonlinear registration. ${ }^{16}$ A mean FA image and a mean FA skeleton were created, which corresponded to the centers of all tracts common to the group (On-line Fig 1). The white matter skeleton is, therefore, a representation of white matter tract geometry, and fiber bundle centers are represented in the mean skeleton. The threshold of the mean FA skeleton white matter mask (shown as a green underlay) was set to an FA value between 0.2 and 0.8 to exclude voxels that consisted of gray matter or CSF. The voxel size was set to $1 \times 1 \times 1 \mathrm{~mm}$ Montreal Neurological Institute space. The obtained binary skeleton mask determined all subsequent processing steps.

FA values of each subject were then projected onto the mean FA skeleton. A voxelwise cross-subject statistical analysis was performed to identify FA differences between patients and healthy control subjects. Group differences were determined by using Randomize, Version 2.9 (The Mathworks, Natick, Massachusetts) (permutation-based nonparametric testing, 5000 permuta- 


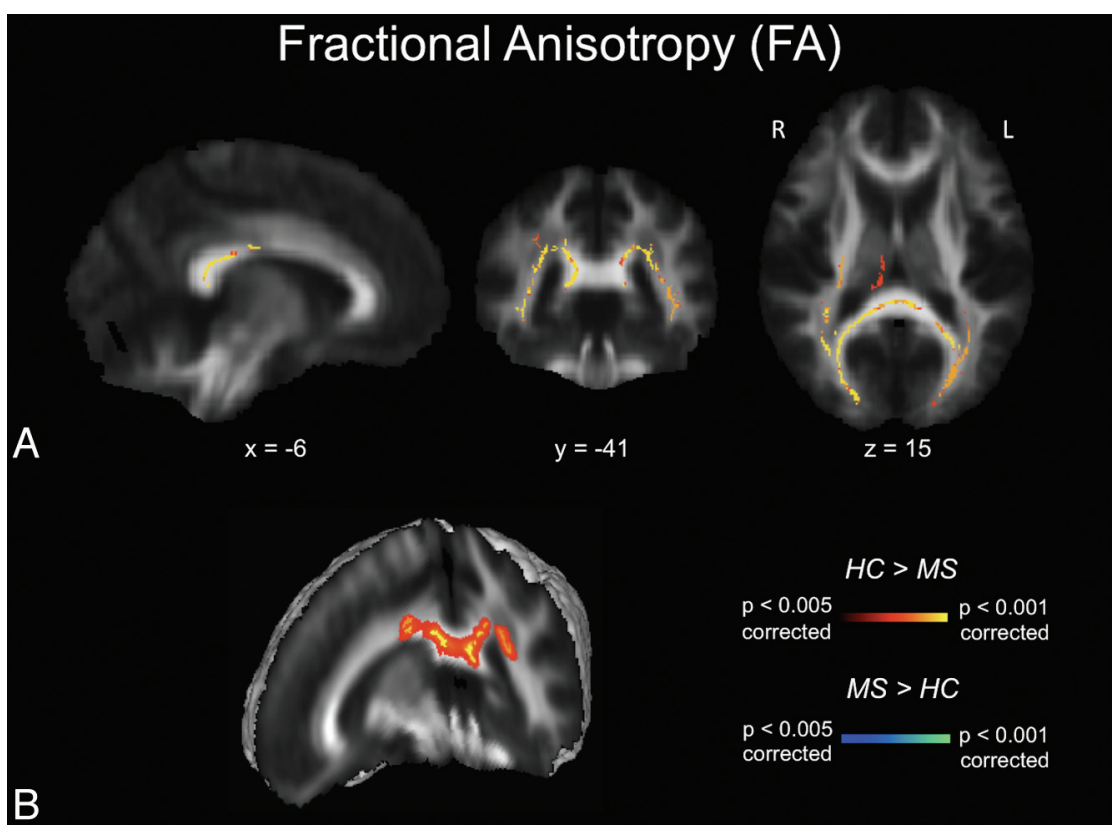

FIG 1. FA maps. A, TBSS FA results for healthy subjects compared with patients with MS. Significant clusters of decreased FA values in patients with MS compared with healthy controls are shown as corresponding $P$ values in red-orange (scale ranging from red to yellow for the comparison $\mathrm{HC}>M S$ and scale ranging from blue to light blue for the comparison MS $>\mathrm{HC}$ ) and have been thresholded at $P<.005$ for between-group comparisons (corrected for multiple comparisons). $B, 3 D$ visualization of significantly ( $P<.01$, corrected for multiple comparisons) different white matter clusters between the 2 groups. Note that the results are thickened for visual purposes only. L indicates left; R, right (radiologic convention); HC, healthy controls.
Illinois), and a $P$ value $<.05$ was considered statistically significant.

\section{RESULTS}

\section{Demographic Data, Disease, and MR Imaging Characteristics}

Fourteen consecutive pediatric patients with MS and age- and sex-matched healthy subjects were included in the study (On-line Table 1). Disease duration in patients ranged between 2 and 57 months (median, 16.5 months). Patients had a low level of neurologic impairment measured by the Kurtzke Expanded Disability Status Scale (mean, $0.75 \pm 1.2$ ) and the Timed 25-Foot Walk Test (3.73 \pm 1.3 seconds), consistent with the relatively short disease duration. Cognitive screening with the Trail-Making Test A (mean, $5.9 \pm 0.5$ ), Trail-Making Test B (mean, $5.72 \pm 0.6$ ), and the Verbal Fluency test (mean, $4.18 \pm$ $0.6)$ showed no differences in normative data $(z$ scores $<2){ }^{21}$ Unusually high completion times $(z$ score $>2)$ were only observed for the Nine-Hole Peg Test (dominant hand mean, $19.5 \pm 2.6 \mathrm{sec}$ onds; nondominant hand mean, $19.7 \pm$ 1.3 seconds) compared with normative tions). ${ }^{23}$ We used threshold-free cluster enhancement to avoid choosing an arbitrary initial cluster-forming threshold. This method provides a voxelwise significance ( $P$ value) that is corrected for multiple comparisons. ${ }^{24}$ The $P$ values are fully corrected for multiple comparisons across voxels but only for each white matter mask and only as 1 -tailed $P$ values. Because we used 4 different white matter masks and investigated betweengroup increases and between-group decreases, a $P<.05$ equaled an adjusted $P<.00625[0.05 /(4 \times 2)]$, a $P$ value $<.01$ corresponded to an adjusted $P<.00125$, a $P<.005$ corresponded to an adjusted $P<.000625$, and a $P<.001$ corresponded to an adjusted $P<.000125$. We considered a $P<.05$ statistically significant.

The same procedure was applied to $\mathrm{MD}, \mathrm{RD}$, and $\mathrm{AD}$ data.

\section{Clinical Correlations}

Within the patient group, clinical results were correlated with mean FA values and of 2 ROIs, which displayed the most significant group differences in the TBSS analysis. The defined ROIs had a diameter of $20 \mathrm{~mm}$ around the peak voxel on the FA skeleton. For all analyses, $P$ values $<.05$ were considered statistically significant.

\section{Statistical Analysis}

All clinical scores were calculated as $z$ scores. A z score lying outside the $95 \%$ normal distribution is conventionally considered abnormal.

The Pearson product moment correlation coefficient $(r)$ was calculated for the correlation between clinical data and mean individual FA values. Correlations were calculated by using the Statistical Package for the Social Sciences, Version 19 (SPSS, Chicago, data. ${ }^{17}$ Mean lesion load for all patients was low in supratentorial $(3.25 \pm 0.96)$ and infratentorial $(2.5 \pm 0.76)$ regions.

\section{Diffusion Parameters in TBSS Analysis}

Mean FA values of the white matter skeleton were significantly decreased in patients with MS compared with age- and sexmatched controls $(P<.005)$. MD $(P<.001), \mathrm{RD}(P<.001)$, and $\mathrm{AD}(P<.05)$ values were increased in patients with MS, with changes in RD being more extensive than those in AD. Detailed results of TBSS analysis can be found in On-line Table 2. The most pronounced decrease in FA was found in the splenium of the corpus callosum $(P<.001)$ (Fig 1$)$. This result remained stable after excluding 3 patients with lesions within the splenium and surrounding regions $(P<.001)$. An additional reduction of FA values was identified in the right temporal and right and left parietal regions (Fig $1 A$ ). We did not detect any FA value increase in the patient group compared with the control group.

Diffusivity maps revealed a widespread increase in MD in most white matter tract regions, including the corpus callosum, superior longitudinal fasciculus, fornix, corona radiata, corticospinal tract, and uncinate fasciculus (Fig 2). Of the 2 components contributing to $\mathrm{MD}, \mathrm{AD}$ increase (Fig 3) was less widespread than RD increase (Fig 4). Neither the anterior or posterior corpus callosum nor the internal capsules exhibited any AD alterations (Fig 3). The anterior internal capsule did not show any RD alterations. We did not detect any significant $\mathrm{MD}, \mathrm{RD}$, or $\mathrm{AD}$ decreases in the patient group.

\section{Correlation between FA and Clinical Scores}

We found a statistically significant correlation between mean skeletal FA and disease duration $(r=-0.57 ; P<.01)$, right temporal $(r=$ 


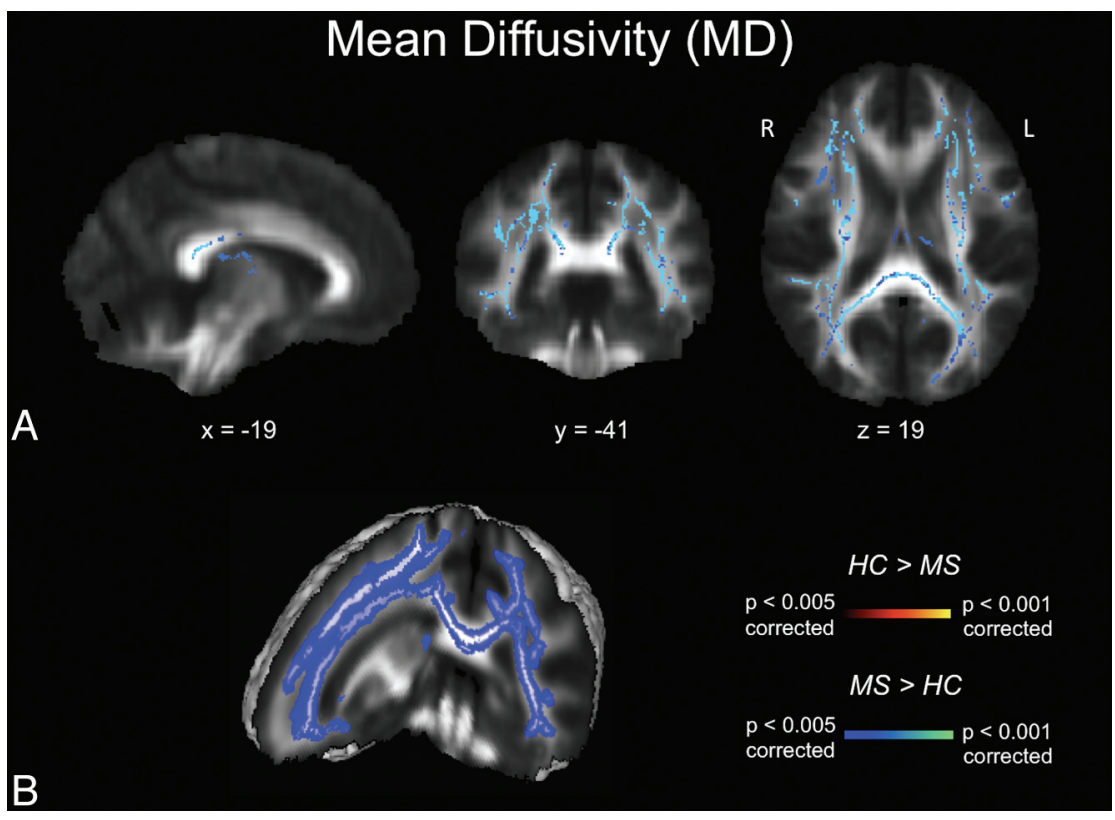

FIG 2. MD maps. A, TBSS MD results for healthy subjects compared with patients with MS. Significant clusters of increased MD values in patients with MS compared with healthy controls are shown as corresponding $P$ values in red-orange (scale ranging from red to yellow for the comparison $\mathrm{HC}>\mathrm{MS}$ and scale ranging from blue to light blue for the comparison MS $>\mathrm{HC}$ ) and have been thresholded at $P<.005$ for between-group comparisons (corrected for multiple comparisons). $B, 3 D$ visualization of significantly $(P<.01$, corrected for multiple comparisons) different white matter clusters between the 2 groups. Note that the results are thickened for visual purposes only. L indicates left; R, right (radiologic convention); HC, healthy controls.

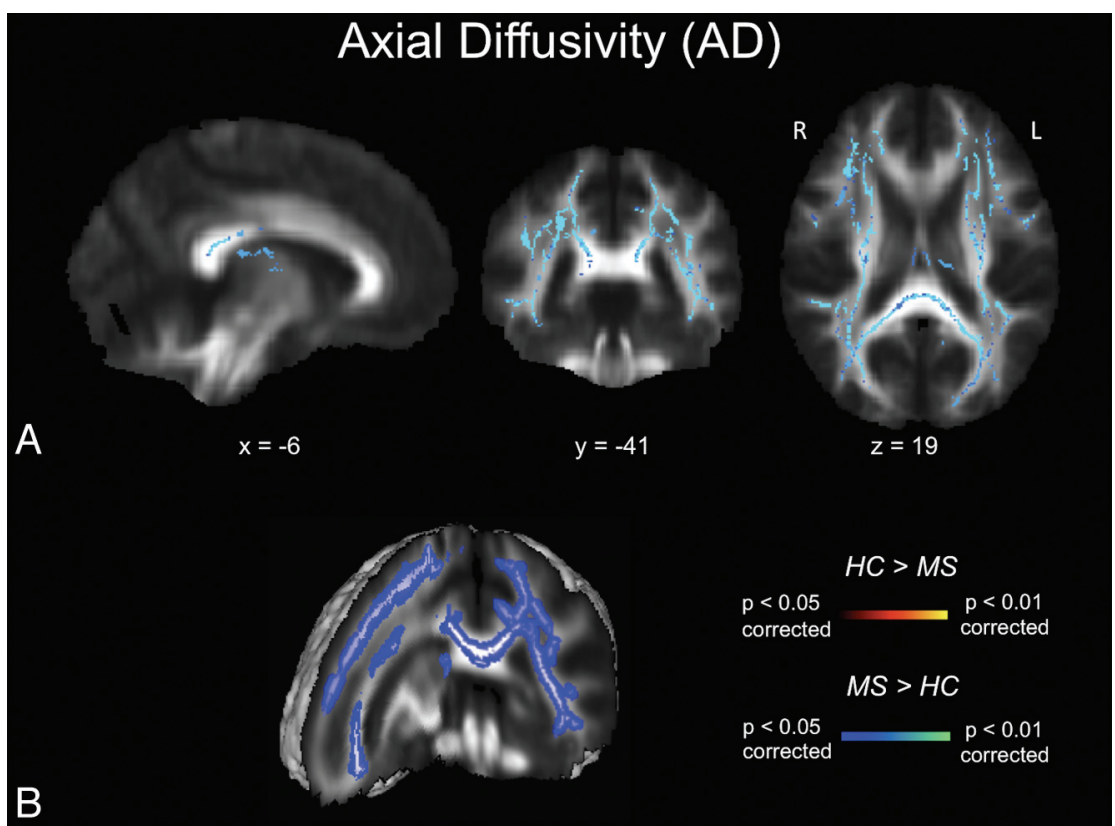

FIG 3. $A D$ maps. A, TBSS AD results for healthy subjects compared with patients with $M S$. Significant clusters of increased AD values in patients with MS compared with healthy controls are shown as corresponding $P$ values in red-orange (scale ranging from red to yellow for the comparison $\mathrm{HC}>\mathrm{MS}$ and scale ranging from blue to light blue for the comparison MS > $\mathrm{HC}$ ) and have been thresholded at $P<.05$ for between-group comparisons (corrected for multiple comparisons). $B, 3 D$ visualization of significantly $(P<.01$, corrected for multiple comparisons) different white matter clusters between the 2 groups. Note that the results are thickened for visual purposes only. L indicates left; R, right (radiologic convention); HC, healthy controls.
$-0.55 ; P<.05)$, and left parietal $(r=$ $-0.54 ; P<.05)$ regions. No significant correlations were found for the Kurtzke Expanded Disability Status Scale, annual relapse rate, number of total relapses, lesion load, Nine-Hole Peg Test, Timed 25-Foot Walk Test, or cognitive screening with FA values.

\section{DISCUSSION}

We investigated the white matter microstructure in children with MS. TBSS analysis revealed significantly decreased FA values along with a widespread increase in $\mathrm{MD}, \mathrm{RD}$, and $\mathrm{AD}$ in patients with MS. These findings provide evidence for the influence of MS in nonlesional macroscopically unaffected WM even at an early stage of the disease. Potential causes of these alterations are diverse and include direct disease effects such as axonal injury and demyelination or indirect effects such as protracted or inhibited maturation.

Our findings are in accordance with previously published data on the microstructure of white matter in childhood MS. ${ }^{12,13}$ Two recent studies of Vishwas et $\mathrm{al}^{14,15}$ demonstrated higher MD and lower mean FA values in all white matter pathways in pediatric patients with MS compared with controls. However, no changes were observed in 27 patients with a single demyelinating event, ${ }^{15}$ from which the authors concluded that there might be a window of opportunity to prevent further damage between the period of a single demyelination and the diagnosis of MS. This is in accordance with the significant negative correlation between mean FA of the white matter skeleton and disease duration that we observed in our study. In adults, conflicting data on the correlation of FA measures with the annual relapse rate, number of total relapses, and lesion load exist. Disease durations of $>3$ years have been reported to negatively correlate with $\mathrm{FA},{ }^{25,26}$ whereas in clinically isolated syndrome, no correlation was reported. ${ }^{6}$

It has been proposed that global changes in white matter occur to a lesser degree in childhood at the beginning of the disease compared with adult-onset MS. ${ }^{6}$ However, early changes in childhood MS are known to be characterized by pathologic MR imaging findings of gray 


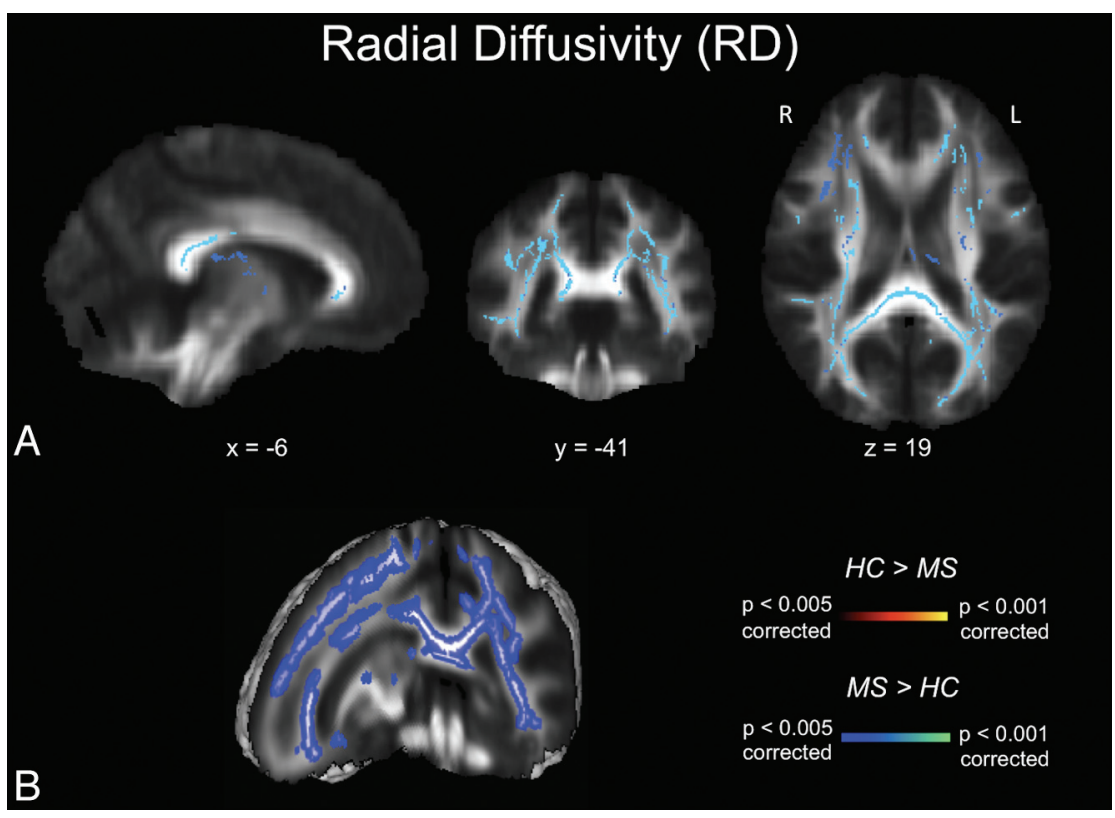

FIG 4. RD maps. A, TBSS RD results for healthy subjects compared with patients with MS. Significant clusters of increased RD values in patients with MS compared with healthy controls are shown as corresponding $P$ values in red-orange (scale ranging from red to yellow for the comparison $\mathrm{HC}>M S$ and scale ranging from blue to light blue for the comparison MS $>\mathrm{HC}$ ) and have been thresholded at $P<.005$ for between-group comparisons (corrected for multiple comparisons). $B, 3 D$ visualization of significantly ( $P<.01$, corrected for multiple comparisons) different white matter clusters between the 2 groups. Note that the results are thickened for visual purposes only. L indicates left; R, right (radiologic convention); HC, healthy controls.

and white matter structures that are generally more subtle compared with MR imaging findings in adult MS. ${ }^{12,13,27}$ This difference may explain why patients with early-onset MS tend to have a longer duration of illness before reaching a state of irreversible disability. ${ }^{28}$ Considering the fact that myelination continues well into adolescence, it is conceivable that MS may interfere with this process of physiologic myelination in addition to the ongoing demyelination by the disease process itself. ${ }^{8,29}$ Longitudinal assessment might be a useful tool for gaining insight into the dynamics of the disease process, especially because established markers for disease activity such as lesion load correlate only moderately with clinical outcome in longitudinal studies in adults and children. ${ }^{2}$

The spatial extent and distribution of increase in diffusivity indices $(\mathrm{MD} / \mathrm{RD} / \mathrm{AD})$ was far more widespread than the decrease in FA values in our patient cohort. Changes in diffusivity parameters are indicators of mainly extracellular diffusion and might be related to axon or myelin damage. Water diffusion in the white matter of the brain is highly sensitive to alterations in the microstructural integrity of cellular membranes; thus, water diffusion is a sensitive marker for early differences in tissue properties related to neuroinflammation, for example. ${ }^{4,5}$ An increase in RD is proposed to reflect myelin destruction as demonstrated in mouse models or human postmortem brain studies. ${ }^{30-32}$ The relevance of $\mathrm{AD}$ as a marker of axonal damage is less well-understood. In our study, $\mathrm{AD}$ was increased but to a lesser extent than $\mathrm{RD}$; these findings are in accordance with those in earlier studies in adults with MS. ${ }^{10,25}$ In contrast, Lin et $\mathrm{al}^{33}$ showed a trend toward lower $\mathrm{AD}$ in the normal-appearing pyramidal tract in adult patients with MS. Recent pathologic studies in degenerative neurologic diseases indicate a topographic concordance between WM loss and $\mathrm{AD}$ changes in Huntington disease or Friedreich ataxia. ${ }^{34,35}$ Ro- sas et al ${ }^{34}$ suggested that the increase in $\mathrm{AD}$ might be due to reduced axonal diameters, resulting in increased extra-axonal space. Thus, interpretation of this diffusivity value is still controversial, with most studies in MS pointing toward an increase in $\mathrm{AD}$. On the other hand, RD plays a predominant role in detecting subtle pathologic damage from very early on in MS, making it a potential marker for monitoring changes in the course of the disease.

In addition to the already-known global decrease in skeletal FA, ${ }^{12}$ patients with MS displayed a regional FA reduction in the splenium of the corpus callosum and in the right temporal and left and right parietal regions. Reduction in FA was most profound in the splenium. These changes in FA may serve as an early indicator of MS white matter pathology in childhood MS. Our results are in accordance with DTI studies in adult-onset MS, which report that the earliest changes in white matter tracts occurred in the corpus callosum. ${ }^{6} \mathrm{~A}$ focal FA decrease in the splenium of the corpus callosum has furthermore been described in patients with isolated optic neuritis. ${ }^{36}$ Our results suggest that the corpus callosum is one of the most affected regions in earlyonset MS. It may, therefore, be a potential target region for the early detection of disease processes by MR imaging. Special attention was drawn to this region by Hagmann et al, ${ }^{37}$ who combined modern MR imaging techniques with network analyses. This publication presented evidence for the existence of a structural core of highly interconnected brain regions, located primarily in the posterior medial and parietal cortex. The splenium of the corpus callosum has been proposed as an integral part of this core, interconnecting both hemispheres.

In our relatively small patient cohort, we observed a significant negative correlation between mean FA of the white matter skeleton and disease duration, while no correlation was found for other clinical parameters. In adults, conflicting data on the correlation of FA measures with annual relapse rate, number of total relapses, and lesion load exist. Disease duration of $>3$ years is reported to negatively correlate with $\mathrm{FA},{ }^{25,26}$ whereas in clinically isolated syndrome, no correlation was reported. ${ }^{6}$

\section{Study Limitations}

There are several limitations to our study that need to be taken into account when interpreting the data. First, the sample sizes in our patient and control cohorts are relatively small due to the rare nature of MS in children. Nevertheless, the alterations in diffusivity parameters were highly significant and appear robust in light of the tests applied. In addition, the MR images of the age- and sex-matched healthy controls were recruited from a local data base, in which the clinical assessment did not include the Nine-Hole Peg Test and the Timed 25-Foot Walk Test. The study-related MR imaging protocol and the MR imaging scanner were identical in both cohorts, though. 


\section{CONCLUSIONS}

Our study demonstrates the white matter microstructure of nonlesional tissue to be affected in childhood MS, even in early stages of the disease. Decreased FA values and increased diffusion parameters may indicate impaired myelination. The observed decrease in FA in the white matter correlated with disease duration.

\section{ACKNOWLEDGMENTS}

We thank all participants in this study.

Disclosures: Astrid Blaschek-RELATED: Grant: Research grant from Merck Serono GmbH;* UNRELATED: Payment for Lectures (including service on speaker bureaus): Speaker honoraria from Biogen Idec $\mathrm{GmbH}$, Merck Serono $\mathrm{GmbH}$, and Bayer Schering GmbH;* Travel/Accommodations/Meeting Expenses Unrelated to Activities Listed: Bayer Schering GmbH, Merck Serono GmbH. Sophia Mueller-UNRELATED: Travel/ Accommodations/Meeting Expenses Unrelated to Activities Listed: I received a travel grant from the GlaxoSmithKline Foundation (2012) to travel to the Annual Meeting of the Organization for Human Brain Mapping. I presented work that is unrelated to the submitted study. Wolfgang Müller-Felber-UNRELATED: Board Membership: Pompe registry board. Florian Heinen—RELATED: Grant: Merck Serono GmbH;* Consulting Fee or Honorarium: Speakers honorarium from Meck Serono; UNRELATED: Payment for Lectures (including service on speaker bureaus): Merz Pharma, Pfizer Deutschland GmbH, and Allergan GmbH. Birgit Ertl-Wagner-RELATED: Grant: German Research Council; UNRELATED: Board Membership: Philips Healthcare, Bracco, Springer Medical Publisher; Consultancy: Munich Medical International, Philips Healthcare; Grants/Grants Pending: Eli Lilly, ${ }^{*}$ Genentech, ${ }^{\star}$ Geurbet, ${ }^{*}$ Merck Serono; ${ }^{*}$ Payment for Lectures (including service on speaker bureaus): Siemens, Bayer Schering; Payment for Manuscript Preparation: Siemens, Springer Medical Publisher, Thieme Medical Publisher, Bracco; Royalties: Springer Medical Publisher, Thieme Medical Publisher; Payment for Development of Educational Presentations: Siemens, Bracco, Springer, Thieme; Stock/Stock Options: Siemens (stock owned by spouse); Travel/Accommodations/Meeting Expenses Unrelated to Activities Listed: Siemens. *Money paid to institution.

\section{REFERENCES}

1. Banwell B, Shroff M, Ness JM, et al. MRI features of pediatric multiple sclerosis. Neurology 2007;68(16 suppl 2):S46-53

2. Mikaeloff Y, Adamsbaum C, Husson B, et al. MRI prognostic factors for relapse after acute CNS inflammatory demyelination in childhood. Brain 2004;127(pt 9):1942-47

3. van der Valk P, Amor S. Preactive lesions in multiple sclerosis. Curr Opin Neurol 2009;22:207-13

4. Pierpaoli C, Basser PJ. Toward a quantitative assessment of diffusion anisotropy. Magn Reson Med 1996;36:893-906

5. Song SK, Sun SW, Ju WK, et al. Diffusion tensor imaging detects and differentiates axon and myelin degeneration in mouse optic nerve after retinal ischemia. Neuroimage 2003;20:1714-22

6. Raz E, Cercignani M, Sbardella E, et al. Clinically isolated syndrome suggestive of multiple sclerosis: voxelwise regional investigation of white and gray matter. Radiology 2010;254:227-34

7. Bodini B, Cercignani M, Khaleeli Z, et al. Corpus callosum damage predicts disability progression and cognitive dysfunction in primaryprogressive MS after five years. Hum Brain Mapp 2013;34:1163-72

8. Barnea-Goraly N, Menon V, Eckert M, et al. White matter development during childhood and adolescence: a cross-sectional diffusion tensor imaging study. Cereb Cortex 2005;15:1848-54

9. Koerte I, Heinen F, Fuchs T, et al. Anisotropy of callosal motor fibers in combination with transcranial magnetic stimulation in the course of motor development. Invest Radiol 2009;44:279-84

10. Roosendaal SD, Geurts JJ, Vrenken H, et al. Regional DTI differences in multiple sclerosis patients. Neuroimage 2009;44:1397-403

11. Utsunomiya H. Diffusion MRI abnormalities in pediatric neurological disorders. Brain Dev 2011;33:235-42

12. Tortorella P, Rocca MA, Mezzapesa DM, et al. MRI quantification of gray and white matter damage in patients with early-onset multiple sclerosis. J Neurol 2006;253:903-07

13. Mezzapesa DM, Rocca MA, Falini A, et al. A preliminary diffusion tensor and magnetization transfer magnetic resonance imaging study of early-onset multiple sclerosis. Arch Neurol 2004;61:366-68

14. Vishwas MS, Chitnis T, Pienaar R, et al. Tract-based analysis of cal- losal, projection, and association pathways in pediatric patients with multiple sclerosis: a preliminary study. AJNR Am J Neuroradiol 2010;31:121-28

15. Vishwas MS, Healy BC, Pienaar R, et al. Diffusion tensor analysis of pediatric multiple sclerosis and clinically isolated syndromes. AJNR Am J Neuroradiol 2013;34:417-23

16. Smith SM, Jenkinson M, Johansen-Berg H, et al. Tract-based spatial statistics: voxelwise analysis of multi-subject diffusion data. Neuroimage 2006;31:1487-505

17. Poole JL, Burtner PA, Torres TA, et al. Measuring dexterity in children using the nine-hole peg test. J Hand Ther 2005;18:348-51

18. Nieuwenhuis MM, Van Tongeren H, Sorensen PS, et al. The six spot step test: a new measurement for walking ability in multiple sclerosis. Mult Scler 2006;12:495-500

19. Reitan RM, Wolfson D. The trail making test as an initial screening procedure for neuropsychological impairment in older children. Arch Clin Neuropsychol 2004;19:281-88

20. Horn W. Leistungsprüfungssystem Manual. Göttingen, Germany: Hogrefe; 1983

21. Helmstaedter C, Schoof K, Rossmann T, et al. Introduction and first validation of EpiTrack Junior, a screening tool for the assessment of cognitive side effects of antiepileptic medication on attention and executive functions in children and adolescents with epilepsy. Epilepsy Behav 2010;19:55-64

22. Smith SM. Fast robust automated brain extraction. Hum Brain Mapp 2002;17:143-55

23. Nichols TE, Holmes AP. Nonparametric permutation tests for functional neuroimaging: a primer with examples. Hum Brain Mapp 2002;15:1-25

24. Smith SM, Nichols TE. Threshold-free cluster enhancement: addressing problems of smoothing, threshold dependence and localisation in cluster inference. Neuroimage 2009;44:83-98

25. Liu Y, Duan Y, He Y, et al. Whole brain white matter changes revealed by multiple diffusion metrics in multiple sclerosis: a TBSS study. Eur J Radiol 2012;81:2826-32

26. Yu HJ, Christodoulou C, Bhise V, et al. Multiple white matter tract abnormalities underlie cognitive impairment in RRMS. Neuroimage 2012;59:3713-22

27. Balássy C, Bernert G, Wober-Bingol C, et al. Long-term MRI observations of childhood-onset relapsing-remitting multiple sclerosis. Neuropediatrics 2001;32:28-37

28. Renoux C, Vukusic S, Mikaeloff Y, et al. Natural history of multiple sclerosis with childhood onset. $N$ Engl J Med 2007;356:2603-13

29. Wilke M, Krageloh-Mann I, Holland SK. Global and local development of gray and white matter volume in normal children and adolescents. Exp Brain Res 2007;178:296-307

30. Song SK, Yoshino J, Le TQ, et al. Demyelination increases radial diffusivity in corpus callosum of mouse brain. Neuroimage 2005;26: 132-40

31. Schmierer K, Wheeler-Kingshott CA, Tozer DJ, et al. Quantitative magnetic resonance of postmortem multiple sclerosis brain before and after fixation. Magn Reson Med 2008;59:268-77

32. Zollinger LV, Kim TH, Hill K, et al. Using diffusion tensor imaging and immunofluorescent assay to evaluate the pathology of multiple sclerosis. J Magn Reson Imaging 2011;33:557-64

33. Lin F, Yu C, Jiang T, et al. Diffusion tensor tractography-based group mapping of the pyramidal tract in relapsing-remitting multiple sclerosis patients. AJNR Am J Neuroradiol 2007;28:278-82

34. Rosas HD, Lee SY, Bender AC, et al. Altered white matter microstructure in the corpus callosum in Huntington's disease: implications for cortical “disconnection.” Neuroimage 2010;49:2995-3004

35. Pagani E, Ginestroni A, Della Nave R, et al. Assessment of brain white matter fiber bundle atrophy in patients with Friedreich ataxia. $R a$ diology 2010;255:882-89

36. Bester M, Heesen C, Schippling S, et al. Early anisotropy changes in the corpus callosum of patients with optic neuritis. Neuroradiology 2008;50:549-57

37. Hagmann P, Cammoun L, Gigandet X, et al. Mapping the structural core of human cerebral cortex. PLoS Biol 2008;6:e159 